\title{
EcoDopplercardiografia em coelhos: uso de midazolam e midazolam associado à cetamina
}

\author{
[Echocardiographic evaluation in rabbits: association of midazolam and ketamine] \\ E.F. Silva ${ }^{1}$, L.R. Borboleta ${ }^{2}$, T.C. Telles ${ }^{2}$, V.B. Fonseca ${ }^{3}$, M.M. Melo ${ }^{4}$ \\ ${ }^{1}$ Aluno de pós-graduação - Escola de Veterinária - UFMG - Belo Horizonte, MG \\ ${ }^{2}$ Aluno de graduação - Escola de Veterinária - UFMG - bolsista de Iniciação Científica \\ ${ }^{3}$ Escola de Veterinária - UFMG \\ Caixa Postal 567 \\ 30123-970 - Belo Horizonte, MG
}

\begin{abstract}
RESUMO
O uso de animais como modelos experimentais muitas vezes exige a administração de sedativos ou anestésicos, particularmente quando se trata de avaliação ecoDopplercardiográfica de coelhos. No entanto, existem poucas informações sobre os protocolos e seus efeitos nestes parâmetros. Diante deste contexto, foram utilizados 20 coelhos Nova Zelândia machos, com cinco meses e 3,2kg, distribuídos em dois grupos de 10 animais cada: G1 - maleato de midazolam associado ao cloridrato de cetamina, e G2 maleato de midazolam. Compararam-se o efeito dos dois protocolos sob os índices funcionais do ventrículo esquerdo e os fluxos valvares, e observaram-se menores valores de frequência cardíaca e da fração de ejeção e maiores valores de diâmetro do ventrículo esquerdo na sístole, de volume sistólico final do ventrículo esquerdo e de diâmetro aórtico no grupo que recebeu apenas maleato de midazolam (G2). Concluiu-se que, o maleato de midazolam apresentou-se mais eficaz, pois causou boa sedação nos animais, permitindo a realização de ecoDopplercardiogramas de qualidade e efeitos limitados no sistema cardiovascular.
\end{abstract}

Palavras-chave: coelho, avaliação ecoDoppercardiográfica, drogas sedativas

\begin{abstract}
The use of animals as experimental models often requires anesthesia, particularly when it comes to echocardiographic evaluation of rabbits. However, there is little information regarding the protocols and their effects on echocardiographic parameters. We used 20 male five month old New Zealand rabbits, weighing $3.2 \mathrm{~kg}$, distributed in two groups of 10 animals each: $\mathrm{G} 1$ - midazolam maleate associated with ketamine hydrochloride, and G2 - midazolam maleate. We compared the effect of two protocols on functional indicators of the left ventricle and the flow valves and we obtained lower values of heart rate and left ventricular ejection fraction and higher values of left ventricular diameter in systole, end-systolic volume of left ventricle and aortic diameter in the group that received only midazolam. Midazolam maleate was more effective because it promoted good sedation, allowing a good quality examination and limited effects on the cardiovascular system.
\end{abstract}

Keywords: rabbit, echoDopplercardiography, sedative drugs

\section{INTRODUÇÃO}

A ecoDopplercardiografia é um método não invasivo útil para a avaliação in vivo das dimensões e funções ventriculares em

Recebido em 17 de agosto de 2010

Aceito em 28 de setembro de 2011

E-mail: marilia@vet.ufmg.br procedimentos clínicos e experimentais, tanto em humanos como em animais (Fontes-Sousa et al., 2009).

O desenvolvimento recente de modelos animais de doenças cardiovasculares fez deles uma 
ferramenta cada vez mais importante em pesquisas (Stypmann et al., 2007). Os coelhos são um importante modelo para pesquisas cardiovasculares devido ao seu pequeno porte, mas grande o suficiente para realizar todos os testes fisiológicos (Muders e Elsner, 2000).

Todavia, o exame de coelhos conscientes é muito difícil, consome muito tempo, necessita de treinamento especial, sobretudo com animais de pesquisa ou animais menos acostumados à manipulação (Schaefer et al., 2005; Stypmann et al., 2007). Coelhos não anestesiados e não acostumados à manipulação sofrem aumento da frequência cardíaca (FC) devido à estimulação simpática em resposta ao estresse (Hobbs et al., 1991).

Vários agentes anestésicos são utilizados para proporcionar suficiente sedação e imobilização, possibilitando a avaliação ecocardiográfica (Vatner e Braunwald, 1975, Lopes et al., 2009). O uso de ansiolíticos evita o aumento expressivo da FC e diminui a dispneia, causados pela agitação e pelo estresse, normalizando o padrão respiratório e diminuindo os artefatos durante o exame (Silva et al., 2008). Valores fisiológicos mais baixos da FC facilitam a mensuração ecocardiográfica (Pennock et al., 1997) e geram imagens melhores, de acordo com a velocidade de processamento de imagem de cada aparelho.

No entanto, sabe-se que estes agentes têm efeitos sobre a função cardíaca (Vatner e Braunwald, 1975; Schaefer et al., 2005; Fontes-Sousa et al., 2009) e que sua extensão depende do tipo de anestesia utilizada (Schaefer et al., 2005; FontesSousa et al., 2009). Roth et al. (2002) mostraram que o tipo de anestesia e o tempo das medidas ecocardiográficas após a aplicação dos agentes anestésicos influenciam significativamente a função cardíaca sistólica.

Stypmann et al. (2007) observaram que não há diferenças nas mensurações estruturais entre coelhos anestesiados ou sem anestesia, porém eles encontraram diferenças entre os parâmetros funcionais avaliados no modo-M e Doppler pulsado. Particularmente, a fração de encurtamento apresentou-se menor em coelhos anestesiados com cetamina-xilazina. Valores de referência para vários parâmetros no modo-M, Doppler e Doppler tecidual foram reportados em coelhos conscientes e em diferentes condições anestésicas (Gan et al., 2004; Fontes-Sousa et al., 2006; Stypmann et al., 2007; Fontes-Sousa et al., 2009). Apesar de a ecoDopplercardiografia ser amplamente utilizada, apenas escassos dados utilizando coelho estão disponíveis em literatura (Stypmann et al., 2007), e poucos abordam os efeitos do protocolo anestésico-sedativo sobre os parâmetros ecoDopplercardiográficos.

Alguns autores utilizaram cloridrato de cetamina $(20 \mathrm{mg} / \mathrm{kg}$ ) associado ao maleato de midazolam $(2 \mathrm{mg} / \mathrm{kg})$ via subcutânea (SC) e cloridrato de cetamina associado a $\alpha$-2-agonistas (xilazina ou medetomidina) como protocolo anestésico para mensuração dos índices ecoDopplercardiográficos em coelhos (FontesSousa et al., 2006; Stypmann et al., 2007; Fontes-Sousa et al., 2009).

Para avaliar estes efeitos, foram escolhidos dois protolocos anestésico-sedativos que promovem imobilização adequada e em tempo hábil para o procedimento ecoDopplercardiográfico e comparou-se um protocolo recentemente utilizado: midazolam associado ao cloridrato de cetamina (Fontes-Sousa et al., 2009) com a utilização apenas do midazolam, ainda não utilizado em pesquisas ecoDopplercardiográficas em coelhos, com a justificativa de este último promover boa sedação e causar menos efeitos cardiovasculares em doses que variam de 0,5 a $1,0 \mathrm{mg} / \mathrm{kg}$ por via intramuscular (IM) (Reusch, 2005).

\section{MATERIAL E MÉTODOS}

Foram utilizados 20 coelhos (Oryctolagus cuniculus cuniculus) adultos, machos, Nova Zelândia branco, com peso médio de $3,2 \mathrm{~kg}$ e cinco meses de idade. Estes animais foram alojados em gaiolas metálicas individuais 90x90x40cm, recebendo água e ração comercial para coelhos (Nature Multivita - Socil Evialis®) ad libidum, em sala arejada, com temperatura entre $22-25^{\circ} \mathrm{C}$, e umidade relativa entre $60-80 \%$. Os coelhos não apresentavam sinais de doença cardiovascular e/ou respiratória, de acordo com o exame físico e ausculta cardiopulmonar cuidadosa, além da ecoDopplercardiografia. Os animais tiveram suas massas corporais devidamente mensuradas antes do protocolo anestésico. 
Os coelhos foram divididos em dois grupos diferentes, com 10 animais cada. $\mathrm{O}$ grupo 1 (G1) recebeu uma associação de cloridrato de cetamina (Dopalem ${ }^{\circledR}$ - Cetamina - Vetbrands, São Paulo, Brasil) $(20 \mathrm{mg} / \mathrm{kg})$ e maleato de midazolam (Dormire ${ }^{\circledR}$ - Midazolam 15mg $(5 \mathrm{mg} / \mathrm{mL})$ - Cristália Produtos Químicos Farmacêuticos - São Paulo, SP) $(2 \mathrm{mg} / \mathrm{kg})$ por via subcutânea, e o grupo 2 (G2) recebeu apenas maleato de midazolam $(1 \mathrm{mg} / \mathrm{kg})$ por via intramuscular. Os exames ecoDopplercardiográficos foram realizados 10min após a administração dos sedativos. Para minimizar efeitos de estresse, os coelhos foram manipulados sempre pelas mesmas pessoas, sendo que os exames foram efetuados em local tranquilo e com os animais com olhos vendados com máscara.

Todos os procedimentos ecoDopplercardiográficos foram realizados conforme descrito por Stypmann et al. (2007) e Fontes-Sousa et al. (2009) para a espécie leporina, os quais seguiram as normas da Sociedade Americana de Ecocardiografia. Foi utilizado o aparelho de ecoDopplercardiografia (Ecocardiógrafo HP Sonos 100 CF - Hewlett Packard) com transdutor setorial multifrequencial de 7,5Mhz. Os animais foram posicionados em decúbito lateral, utilizando-se as regiões paraesternais direita e esquerda, do terceiro ao sexto espaços intercostais, sendo este posicionamento o mais utilizado nas pesquisas com coelhos (Thomas et al., 1993, Pennock et al., 1997; Gan et al., 2004; Fontes-Sousa et al., 2009).

Após tricotomia da região torácica, com o uso de gel condutor para ecocardiografia (Gel condutor para eletro e ecocardiografia Mercur $\left.{ }^{\circledR}\right)$, avaliaram-se os modos bidimensional, $\mathrm{M}$, Doppler pulsado e por mapeamento de fluxo em cores, método descrito por Stypmann et al. (2007), realizando-se três mensurações de cada variável e utilizando-se o valor final de suas médias (Stypmann et al. 2007; Silva et al., 2008).

No modo bidimensional, avaliou-se a morfologia das câmaras cardíacas. No modo $\mathrm{M}$, foram analisadas, no final da sístole e no final da diástole, a dimensão interna do ventrículo esquerdo (VE), espessura do septo interventricular e a espessura da parede livre do ventrículo esquerdo (Fontes-Sousa et al., 2009).
Foram calculados os volumes sistólico e diastólico finais do ventrículo esquerdo, além da fração de ejeção, segundo fórmula de Teicholz (Boon, 1998), que, em vários estudos em humanos e cães, demonstrou grande correlação em mensurações invasivas do débito cardíaco e de encurtamento sistólico do VE. Ainda foi mensurado o diâmetro interno do átrio esquerdo e da artéria aorta. No modo Doppler, avaliaramse os picos de velocidade dos fluxos na valva mitral e na aórtica.

Este estudo foi conduzido de acordo com as regras do Comitê de Ética em Experimentação Animal (protocolo número 187/08).

\section{ANÁLISE ESTATÍSTICA}

O delineamento experimental aplicado foi $\mathrm{O}$ inteiramente ao acaso. Para avaliação de normalidade, foi utilizado o teste de Lilliefors. Os dados referentes aos diferentes grupos e tempos foram colocados em tabelas e analisados pelo programa SISVAR® (SISVAR® - UFLA Brasil) para análise de variância (ANOVA) e para o teste exato de Fischer. Com o objetivo de verificar os diferentes tratamentos sobre os parâmetros ecoDopplercardiográficos, as médias foram comparadas por este teste, com nível de significância de $5 \%(\mathrm{P}<0,05)$.

\section{RESULTADOS E DISCUSSÃO}

Foi observada boa sedação em ambos os protocolos anestésico-sedativos, evidenciando que, em relação à contenção e duração da sedação, os dois protocolos foram eficazes, pois permitiram as mensuraçõespadrão nos 20 animais. As mensurações ecoDopplercardiográficas foram feitas no período de 20 minutos.

Observou-se diferença $(\mathrm{P}<0,05)$ entre as frequências cardíacas (FC) dos animais que receberam os dois protocolos propostos, sendo que os animais que receberam apenas o maleato de midazolam apresentaram as menores FC (Tab. 1). A FC obtida com o protocolo com o maleato de midazolam $(217 \pm 12 \mathrm{bpm})$ foi a que mais se aproximou à FC de coelhos sem restrição, segundo Marano et al. (1996), que mensuraram a FC de coelhos Nova Zelândia por telemetria, sem nenhuma restrição ou medicação, entre $218 \pm 4 \mathrm{bpm}$. 
Tabela 1. Valores ecoDopplercardiográficos de coelhos Nova Zelândia machos obtidos com o protocolo de $2 \mathrm{mg} / \mathrm{kg}$ de maleato de midazolam e $20 \mathrm{mg} / \mathrm{kg}$ de cloridrato de cetamina por via subcutânea $(\mathrm{G} 1)$ e $1 \mathrm{mg} / \mathrm{kg}$ de maleato de midazolam por via intramuscular $(\mathrm{G} 2)$

\begin{tabular}{|c|c|c|c|c|c|}
\hline & Mensuração & $\begin{array}{l}\text { Midazolam e } \\
\text { cetamina }(\mathrm{G} 1)\end{array}$ & $p$ & Midazolam (G2) & $\mathrm{CV}(\%)$ \\
\hline \multirow{13}{*}{$\begin{array}{l}\text { Ventrículo } \\
\text { esquerdo }\end{array}$} & FC (bpm) & $236 \pm 17$ & 0,00913 & $217 \pm 12$ & 6.489 \\
\hline & $\mathrm{dAE}(\mathrm{mm})$ & $10,80 \pm 1,00$ & $* * *$ & $11,00 \pm 1,05$ & $* * *$ \\
\hline & $\mathrm{dAO}(\mathrm{mm})$ & $6,60 \pm 0,70$ & 0,01227 & $7,80 \pm 1,20$ & $* * *$ \\
\hline & $\mathrm{AE} / \mathrm{AO}$ & $1,64 \pm 0,13$ & 0,08233 & $1,47 \pm 0,25$ & 12.848 \\
\hline & $\mathrm{VE}(\mathrm{mL})$ & $2,87 \pm 1,03$ & 0,16153 & $3,57 \pm 1,10$ & 33.022 \\
\hline & SIVd (mm) & $2,57 \pm 0,27$ & 0,20669 & $2,77 \pm 0,40$ & 12.570 \\
\hline & DVEd (mm) & $12,17 \pm 1,90$ & 0,08939 & $13,61 \pm 1,68$ & 13.926 \\
\hline & DVEs (mm) & $6,76 \pm 1,50$ & 0,02182 & $8,23 \pm 1,10$ & 17.483 \\
\hline & PPVEd (mm) & $2,88 \pm 0,52$ & $* * *$ & $2,87 \pm 0,24$ & 14.046 \\
\hline & VSFVE (mL) & $7,76 \pm 3,88$ & 0,01366 & $12,55 \pm 3,97$ & 38.671 \\
\hline & VDEVE (mL) & $3,65 \pm 1,36$ & 0,0718 & $4,82 \pm 1,38$ & 32.405 \\
\hline & $\mathrm{FE}(\%)$ & $79,23 \pm 6,70$ & 0,04947 & $73,69 \pm 4,94$ & 7.697 \\
\hline & $\% \Delta \mathrm{D}(\%)$ & $45,23 \pm 8,24$ & 0,06007 & $40,68 \pm 5,08$ & 15.364 \\
\hline $\begin{array}{l}\text { Fluxo } \\
\text { mitral }\end{array}$ & $V \max (\mathrm{cm} / \mathrm{s})$ & $62,69 \pm 9,67$ & 0,27933 & $67,84 \pm 10,96$ & 15.837 \\
\hline $\begin{array}{l}\text { Fluxo } \\
\text { aorta }\end{array}$ & $V \max (\mathrm{cm} / \mathrm{s})$ & $87,37 \pm 13,71$ & $* * *$ & $92,27 \pm 7,95$ & 12.479 \\
\hline
\end{tabular}

$\overline{\mathrm{FC}}=$ frequência cardíaca; $\mathrm{dAE}=$ diâmetro do átrio esquerdo; $\mathrm{dAO}=$ diâmetro da artéria aorta; $\mathrm{AE} / \mathrm{AO}=$ relação entre átrio esquerdo e artéria aorta; $\mathrm{VE}$ = volume ejetado; $\mathrm{SIVd}=$ espessura do septo interventricular na diástole; DVEd = diâmetro diastólico do VE; DVEs = diâmetro sistólico do VE; PPVEd = espessura da parede livre do VE na diástole; VSFVE = volume sistólico final do VE; VDEVE = volume diastólico final do VE; FE = fração de ejeção; $\% \Delta \mathrm{D}=$ fração de encurtamento; Vmax = velocidade máxima do fluxo.

Os coelhos deste experimento, sedados com a associação maleato de midazolam e cloridrato de cetamina, apresentaram FC de $236 \pm 17 \mathrm{bpm}$, menores que a do estudo de Fontes-Sousa et al. (2009), utilizando o mesmo protocolo $(262 \pm 37 \mathrm{bpm})$. Estes valores foram semelhantes ao de animais não anestesiados, mas acostumados à manipulação (180-250bpm e 234 \pm 26 bpm) (Marano et al., 1996; Stypmann et al., 2007). A utilização de métodos que minimizem a excitação dos animais, como a vedação completa dos olhos com a máscara usada neste estudo, contribui para menores frequências cardíacas e pode explicar a diferença observada. Estes valores foram ainda próximos ao de animais anestesiados com diazepam e pentobarbital $(221 \pm 21 \mathrm{bpm})$ (Gan et al., 2004), porém consideravelmente altos em comparação aos coelhos que receberam cloridrato de cetamina e $\alpha$-2-agonistas (xilazina ou medetomidina) $(155 \pm 29$ e $198 \pm 37 \mathrm{bpm}$, respectivamente) (Fontes-Sousa et al., 2006; Stypmann et al., 2007). Esta diferença deve-se, sobretudo, ao efeito depressor que a xilazina tem sobre a frequência cardíaca, demonstrando que os protocolos de sedação devem evitar esse fármaco. Portanto, prefere-se o uso dos benzodiazepínicos, drogas que alteram menos a frequência cardíaca.

Similarmente a outros mamíferos, a relação átrio esquerdo-aorta (AE/AO) de coelhos é 1,0 a 1,5, o átrio esquerdo (AE) e o direito são comparáveis em tamanho, a aorta (AO) e a artéria pulmonar principal têm diâmetros similares (Pariaut, 2009). Fontes-Sousa et al. (2006) e Fontes-Sousa et al. (2009), encontraram valores da relação AE/AO que corroboram com Pariaut (2009), de $1,15 \pm 0,19$ e $1,17 \pm 0,14$, respectivamente. Neste estudo, a relação AE/AO encontrada foi de $1,47 \pm 0,25$ utilizando-se o protocolo de maleato de midazolam, valor dentro dos considerados normais para a espécie (1 a 1,5), o que mostra que esse benzodiazepínico, quando utilizado isoladamente, não altera parâmetros ecocardiográficos. Já a associação de maleato de midazolam e cloridrato de cetamina mostrou média de 1,64 $\pm 0,13$, ligeiramente acima dos valores normais. $\mathrm{O}$ uso da cetamina pode induzir um aumento do retorno venoso e consequente 
aumento de volume atrial, devido aos seus efeitos nefrogênicos, como estudados por Nocite et al. (1977).

O diâmetro do ventrículo esquerdo durante a sístole (DVEs) e o volume sistólico final do VE (VSFVE) também apresentaram diferença estatística significativa $(\mathrm{P}<0,05)$, sendo que, nos animais que receberam apenas o midazolam, foram verificados valores maiores. Associandose aos maiores valores médios de FC, estes achados corroboram com Nocite et al. (1977), que relataram que, em coelhos, o cloridrato de cetamina eleva a pressão arterial (PA) e a frequência cardíaca (FC), provocando menores valores de volume sistólico, inotropismo cardíaco alterado e variações da resistência vascular periférica.

O grupo que recebeu apenas maleato de midazolam (G2) apresentou valores de DVEs de $8,23 \pm 1,10 \mathrm{~mm}$, que foram comparativamente semelhantes aos valores obtidos por Gan et al. (2004) $(9,5 \pm 0,7 \mathrm{~mm})$, Stypmann et al. (2007) $(10,74 \pm 0,89 \mathrm{em}$ animais anestesiados e $10,09 \pm 0,91 \mathrm{~mm}$ em animais sem anestesia) e Fontes-Sousa et al. (2009) (8,64 $\pm 0,82 \mathrm{~mm})$. O protocolo anestésico de Fontes-Sousa et al. (2006) foi o que resultou em maiores médias do DVEs $(14,37 \pm 1,49 \mathrm{~mm})$. Os animais que receberam midazolam-cetamina tiveram valores do DVEs de $6,76 \pm 1,50 \mathrm{~mm}$, comparativamente menor a todos os protocolos utilizados nesse estudo. Em relação ao VSFVE, os animais que foram sedados apenas com maleato de midazolam apresentaram valores de $12,55 \pm 3,97 \mathrm{~mL}$, e o grupo que recebeu a associação maleato de midazolam e cloridrato de cetamina valores de $7,76 \pm 3,88 \mathrm{ml}$. A cetamina contribui para a elevação da frequência cardíaca e também maior força de contração, aumentado o volume de ejeção, o que explica os menores valores do DVE no final da sístole, assim como menor VSFVE.

A fração de ejeção (FE) foi $7 \%$ menor no grupo que recebeu maleato de midazolam em comparação à associação $(\mathrm{P}<0,05)$, provavelmente devido ao aumento do VE durante a sístole (Stypmann et al., 2007). Para calcular os volumes e a FE, são disponibilizadas fórmulas matemáticas. Neste estudo, foi escolhida a de Teicholz, por se assemelhar mais às mensurações invasivas e apresentar maior correlação nas mensurações em gatos. Stypmann et al. (2007) utilizaram a fórmula de Simpson e Fontes-Sousa et al. (2006; 2009), a fórmula cúbica para a obtenção das FE. Pariaut (2009) não informou a fórmula utilizada, dificultando a comparação com os valores obtidos neste estudo.

Não foi observada depressão cardiorrespiratória nem no G1 (maleato de midazolam mais cloridrato de cetamina) - o que corrobora com Dupras et al. (2001), que alega ser esta mínima nem no G2 (maleato de midazolam), estando de acordo com Reusch (2005).

Porém, a avaliação dos efeitos do cloridrato de cetamina sobre a contratilidade miocárdica é sempre mais difícil. As alterações no estado contrátil estão sempre mascaradas por sua ação no tônus simpático, as quais provocam menores valores de volume sistólico (White et al., 1982), aumentando o inotropismo cardíaco e a resistência vascular periférica (Nocite et al., 1977), alterando pré e pós-carga. Para minimizar os efeitos da variação de pré-carga/pós-carga causados pelos protocolos anestésico-sedativos na avaliação dos seus efeitos sobre a função cardíaca (sistólica e diastólica), recomenda-se o uso de Doppler tecidual. O Doppler tecidual é o método que sofre menor influência de fatores periféricos como pré-carga, pós-carga e frequência cardíaca (Masaki et al., 2006).

\section{CONCLUSÃO}

Concluiu-se que, devido aos efeitos simpatomiméticos do cloridrato de cetamina (crono e inotropismo positivo) na alteração dos parâmetros ecocardiográficos (diâmetro do VE na sístole, volume sistólico final do VE e fração de ejeção), bem como à sua ausência no protocolo utilizando apenas com maleato de midazolam, esse se apresentou mais eficaz com boa sedação. Este protocolo ainda permitiu a realização de exame de qualidade, a estabilização da frequência cardíaca a valores fisiológicos menores e a mínima estimulação simpatomimética que altera as mensurações ecoDopplercardiográficas.

\section{AGRADECIMENTOS}

Ao Hospital Veterinário da Escola de Veterinária da Universidade Federal de Minas Gerais e à Fundação de Amparo a Pesquisa de Minas Ferais (FAPEMIG). 


\section{REFERÊNCIAS BIBLIOGRÁFICAS}

BOON, J.A. Manual of veterinary echocardiography. Baltimore: Williams \& Wilkins, 1998, 478p.

DUPRAS, J.; VACHON, P.; CUVELLIEZ, S.; BALIS, D. Anesthesia of the New Zealand rabbit using the combination of tiletamine-zolazepam and ketamine-midazolam with or without xylazine. Can Vet. J., v.42, p.455-460, 2001.

FONTES-SOUSA, A.P.; BRAS-SILVA, C.; MOURA, C. et al. M-mode and Doppler echocardiographic reference values for male New Zealand white rabbits. Am. J. Vet. Res., v.67, p.1725-1729, 2006.

FONTES-SOUSA, A.P.; MOURA, C.; CARNEIRO, C.S. et al. Echocardiographic evaluation including tissue Doppler imaging in New Zealand white rabbits sedated with ketamine and midazolam. Vet. J., v.181, p.326-331, 2009.

GAN, L.; WIKSTROM, J.; BRANDT-ELIASSON, U.; WANDT, B. Amplitude and velocity of mitral annulus motion in rabbits. Echocardiography: $J . C V$ Ultrasound Allied Tech., v.21, p.313-317, 2004.

HOBBS, B.A.; RAOLHALL, T.G.; SPRENKEL, T.L. et al. Comparison of several combinations for anesthesia in rabbits. Am. J. Vet. Res., v.52, p.669-674, 1991.

LOPES, P.C.F.; NUNES, N.; SOUSA, M.G. et al. Efeitos de diferentes $\mathrm{FiO} 2$ sobre variáveis ecocardiográficas em cães submetidos à infusão contínua de propofol. Arq. Bras. Med. Vet. Zootec., v.61, p.345-352, 2009.

MARANO, G.; GRIGIONI, M.; TIBURZI, F. et al. Effects of isoflurane on cardiovascular system and sympathovagal balance in New Zealand white rabbits. J. Cardiovasc. Pharmacol., v.28, p.513-518, 1996.

MASAKI, N.; ROMAN, K.S.; KINGDON, J. et al. Assessment of the evolution of normal fetal diastolic function during mid and late gestation by spectral Doppler tissue echocardiography. J. Am. Soc. Echocardiog., v.19, p.1431-1437, 2006.

MUDERS, F.; ELSNER, D. Animal models of chronic heart failure. Pharmacol. Res., v.41, p.605-612, 2000.
NOCITE, J.R.; VICHI, F.L.; MAGALDI, A.J.B. Ketamina e hipertensão arterial nefrogênica. Rev. Bras. Anestesiol., v.27, 1977.

PARIAUT, R. Cardiovascular physiology and disease of the rabbit. Vet. Clin. North Am. Exot. Anim. Pract., v.12, p.135-144, 2009.

PENNOCK, G.D.; YUN, D.D.; AGARWAL, P.G. et al. Echocardiographic changes after myocardial infarction in a model of left ventricular diastolic dysfunction. Am. J. Physiol. Heart Circ. Physiol., v.273, p.2018-2029, 1997.

REUSCH, B. Investigation and management of cardiovascular disease in rabbits. In Pract., v.27, p.418-25, 2005.

ROTH, D.M.; SWANEY, J.S.; DALTON, N.D. et al. Impact of anesthesia on cardiac function during echocardiography in mice. Am. J. Physiol. Heart Circ. Physiol., v.282, p.H2134-H2140, 2002.

SCHAEFER, A.; MEYER, G.P.; BRAND, B. et al. Effects of anesthesia on diastolic function in mice assessed by echocardiography. Echocardiography, v.22, p.665-670, 2005.

SILVA, E.F; MELO, M.B.; MUZZI, R.A.L. et al. Índices ecodopplercardiográficos de função ventricular esquerda em cães das raças Boxer e Schnauzer Miniatura. Arq. Bras. Med. Vet. Zootec., v.60, p.71-75, 2008.

STYPMANN, J.; ENGELEN, M.A.; BREITHARDT, A. et al. Doppler echocardiography and tissue doppler imaging in the healthy rabbit: differences of cardiac function during awake and anaesthetised examination. Int. J. Cardiol., v.115, p.164-170, 2007.

THOMAS, W.P.; GABER, C.E.; JACOBS, G.J. et al. Recommendations for standards in transthoracic twodimensional echocardiography in the dog and cat. $J$. Vet. Intern. Med., v.7, p.247-252, 1993.

VATNER, S.F.; BRAUNWALD, E. Cardiovascular control mechanisms in the conscious state. N. Engl. J. Med., v.293, p.70-976, 1975.

WHITE, P.F.; WAY, W.; TREVOR, A.J. Ketamine - its pharmacology and therapeutic uses. Anesthesiology, v.56, p.199-236, 1982. 